\title{
Perfil epidemiológico da leishmaniose visceral no Brasil, no período de 2010 a 2019
}

\author{
Epidemiological profile of visceral leishmaniasis in Brazil, from 2010 to 2019 \\ Perfil epidemiológico de leishmaniasis visceral en Brasil, de 2010 a 2019
}

Ricardo Gois de Lima', Taiane Menezes Mendonça', Thiago da Silva Mendes², Marcos Vinícius Costa Menezes².

\section{RESUMO}

Objetivo: Analisar os indicadores epidemiológicos da leishmaniose visceral em todo o país, no período de 2010 a 2019. Métodos: Os dados foram obtidos do departamento de informática do Sistema Único de Saúde do Brasil (DATASUS) e do Instituto Brasileiro de Geografia de Estatística (IBGE) no período de 2010 a 2019. Resultados: Observou-se um total de 24.257 internações por Leishmaniose Visceral em todo o país, com predomínio na região Nordeste $55,03 \%$. O sexo masculino foi responsável por $62,08 \%$ dos casos. Quanto à idade, a faixa mais acometida foi de 1-4 anos de idade (31,07\%). Foram evidenciados 1021 óbitos e uma média de permanência hospitalar de 13,9 dias. Conclusão: Mantêm-se o maior número de casos na região Nordeste, com maior acometimento em homens e maior incidência em crianças. No entanto, todas as transformações ocorridas em nosso meio acarretam a expansão das áreas endêmicas e aparecimento de novos focos. Por fim, foi constatada uma menor taxa de mortalidade que pode ser atribuído ao diagnóstico mais precoce da doença.

Palavras-chave: Leishmaniose visceral, Prevenção de doenças, Epidemiologia.

\section{ABSTRACT}

Objective: To analyze the epidemiological indicators of visceral leishmaniasis across the country, from 2010 to 2019. Methods: Data were obtained from the IT department of the Brazilian Unified Health System (DATASUS) and from the Brazilian Institute of Geography of Statistics (IBGE) in the period from 2010 to 2019. Results: A total of 24,257 admissions for Leishmaniasis were observed Visceral throughout the country, with a predominance in the Northeast region of $55.03 \%$. The male sex was responsible for $62.08 \%$ of the cases. As for age, the most affected range was $1-4$ years old (31.07\%). There were 1021 deaths and an average hospital stay of 13.9 days. Conclusion: The largest number of cases remains in the Northeast region, with greater involvement in men and greater incidence in children. However, all the changes that have occurred in our environment have led to the expansion of endemic areas and the appearance of new outbreaks. Finally, a lower mortality rate was found that can be attributed to the earlier diagnosis of the disease.

Key words: Visceral leishmaniasis, Prevention of diseases, Epidemiology.

\section{RESUMEN}

Objetivo: Analizar los indicadores epidemiológicos de leishmaniasis visceral en todo el país, de 2010 a 2019. Métodos: Los datos se obtuvieron del departamento de TI del Sistema Único de Salud de Brasil (DATASUS) y del Instituto Brasileño de Geografía de Estadística (IBGE) en el período de 2010 a 2019. Resultados: Se observaron un total de 24,257 ingresos por Leishmaniasis Visceral en todo el país, con predominio en la

${ }^{1}$ Universidade Tiradentes (UNIT), Aracaju - SE. *E-mail: ricardo_gois03@hotmail.com

2 Universidade Federal de Sergipe (UFS), Lagarto - SE. 
región Nordeste del $55,03 \%$. El sexo masculino fue responsable del $62,08 \%$ de los casos. En cuanto a la edad, el rango más afectado fue el de 1 a 4 años (31,07\%). Hubo 1021 muertes y una estancia hospitalaria media de 13,9 días. Conclusión: El mayor número de casos permanece en la región Nordeste, con mayor afectación en hombres y mayor incidencia en niños. Sin embargo, todos los cambios que se han producido en nuestro entorno han provocado la expansión de áreas endémicas y la aparición de nuevos brotes. Finalmente, se encontró una menor tasa de mortalidad que se puede atribuir al diagnóstico precoz de la enfermedad.

Palabras clave: Leishmaniasis visceral, Prevención de enfermedades, Epidemiología.

\section{INTRODUÇÃO}

A Leishmaniose Visceral (LV), também conhecida como calazar, é uma doença parasitária de grande impacto na saúde pública (BRASIL, 2014). É uma doença crônica e sistêmica, que, quando não tratada, tem a chance de evoluir para óbito em mais de $90 \%$ dos casos, registra-se anualmente 59.000 óbitos. Nos últimos dez anos, foram registrados 42.067 casos no Brasil. Diante disso, ocorreram 2.704 óbitos, e a incidência média foi de 1,92 casos por 100.000 habitantes. Seu agente etiológico é o protozoário Leishmania chagasi, e é transmitido pelo vetor Lutzomyia longipalpis (BRASIL, 2014; BRASIL, 2019).

De acordo com a Organização Pan Americana da Saúde (OPAS) possui distribuição mundial em 76 países, sendo endêmica em 12 países das Américas. Cerca de 96\% dos casos nesta região, estão concentrados no Brasil, no entanto, se observa uma expansão geográfica na Argentina, Colômbia, Paraguai e Venezuela (OPAS, 2018).

Em nosso País, a doença é endêmica e surtos frequentes estão sendo registrados. Conforme a OPAS (2018) e o Ministério da Saúde (2019), no início, a Leishmaniose Visceral era limitada a áreas rurais e a pequenas localidades urbanas, hoje, no entanto, já se percebe um crescimento para os grandes centros. Há registro nas 5 regiões brasileiras, com destaque das regiões Nordeste, Sudeste e Centro-Oeste. Além disso, nota-se que a doença apresenta aspectos climáticos, sociais e geográficos diferenciados.

Segundo o Ministério da saúde (2019), na área urbana, o cão (Canis familiaris) é a principal fonte de infecção. A doença canina tem precedido a ocorrência de casos humanos e a infecção em cães tem sido mais prevalente que no homem. No Brasil, a Leishmaniose Visceral Canina (LVC) é considerada mais importante que a doença humana, pois os cães infectados têm alta carga parasitária na pele, sendo considerado a principal fonte de infecção para os vetores (RODRIGUES ACM, et al., 2017).

No ambiente silvestre, os reservatórios são as raposas (Dusicyon vetulus e Cerdocyon thous) e os marsupiais (Didelphis albiventris). O Ministério da Saúde (2014) afirma que a doença no cão é de evolução lenta e início insidioso. A leishmaniose visceral canina é uma doença sistêmica severa cuja manifestações clínicas estão intrinsecamente dependentes do tipo de resposta imunológica expressa pelo animal infectado. Assim, o quadro clínico dos cães infectados apresenta um espectro de características clínicas que varia do aparente estado sadio a um severo estágio final.

Diante dessa problemática e nas palavras de Ministério da saúde (2014), o governo brasileiro desenvolveu o Programa de Controle da Leishmaniose Visceral (PCLV) para o combate da doença, tendo como objetivo reduzir a incidência, mortalidade, letalidade e o grau de morbidade por meio do diagnóstico e tratamento precoce. Além disso, foram criadas estratégias pactuadas para diminuir a transmissão do parasito a fim de controlar a ação dos vetores e reservatórios. Já que a identificação precoce dos pacientes que poderão evoluir com gravidade é de fundamental importância para reduzir a letalidade por meio da instituição de medidas profiláticas e terapêuticas oportunas (RODRIGUES ACM, et al., 2017).

De acordo com o Ministério da Saúde (2019), e Correia AVGM (2015), a suspeita clínica é levantada ainda na fase aguda da doença, onde o paciente apresenta febre com duração inferior a quatro semanas, palidez cutâneo-mucosa e hepatoesplenomegalia, o baço geralmente não ultrapassa a $5 \mathrm{~cm}$ do rebordo costal esquerdo, mas seu estado geral está preservado. Seguindo um quadro arrastado, caracterizado por 
emagrecimento progressivo, palidez cutâneo-mucosa e aumento da hepatoesplenomegalia. A doença pode evoluir para o período final, com febre contínua e comprometimento mais intenso do estado geral. Instala-se então, a desnutrição, edema dos membros inferiores, hemorragias, icterícia e ascite.

Ministério da Saúde (2019), declara que o diagnóstico pode ser feito através da pesquisa de anticorpos contra Leishmania: Imunofluorescência indireta (RIFI), testes rápidos imunocromatográficos, ensaio imunoenzimático (ELISA). É fato que títulos variáveis dos exames sorológicos podem persistir positivos por longo período, mesmo após o tratamento. Assim, o resultado de um teste positivo, na ausência de manifestações clínicas, não autoriza a instituição de terapêutica. Outra forma é o diagnóstico parasitológico, que é de certeza, feito pelo encontro de formas amastigotas do parasito, em material biológico obtido preferencialmente da medula óssea, do linfonodo ou do baço.

Conforme exposto em Ministério da Saúde (2019), quando o diagnóstico sorológico ou parasitológico não estiver disponível ou na demora da sua liberação, o tratamento deve ser prontamente iniciado. É preciso fazer hidratação, antitérmicos, antibióticos, hemoterapia e suporte nutricional. $\mathrm{O}$ antimonial pentavalente tem a vantagem de poder ser administrado no nível ambulatorial, o que diminui os riscos relacionados à hospitalização.

Ainda de acordo com Ministério da Saúde (2019), a anfotericina B é a única opção no tratamento de gestantes e de pacientes que tenham contraindicações ou que manifestem toxicidade ou refratariedade relacionada ao uso dos antimoniais pentavalentes. Em relação aos casos de LV com maior risco de evoluir para óbito, estes devem ser internados e tratados em hospitais de referência, já os casos leves ou intermediários devem ser assistidos no nível ambulatorial, em unidades de saúde com profissionais capacitados.

Assim, para o Ministério da Saúde (2019), é importante fazer o controle de cura através de critérios clínicos, com seguimento ambulatorial do paciente, é importante afirmar que a realização do exame parasitológico, como também, de provas sorológicas, não são necessárias após o final do tratamento.

O Presente estudo tem como objetivo desenvolver e aprofundar o conhecimento sobre a leishmaniose visceral no nosso país, de dimensões continentais, para então podermos aprofundar os métodos de diagnóstico para essa patologia tão prevalente em determinadas regiões do nosso país. Sem contar na otimização da terapêutica atribuída para cada caso, fazendo com que exista uma redução das hospitalizações e custos gerados para o serviço de saúde brasileiro. Além de buscar uma maior compreensão epidemiológica, sobre os seus fatores causais e de prevalência.

\section{MÉTODOS}

As dimensões continentais do Brasil resultam numa ampla variabilidade climática e de biomas a que pertencem as capitais brasileiras e diferenças espaciais quanto o processo de adoecimento. No período de 2010 a 2019, foi feito um estudo sobre os casos de LV internados no país, do tipo epidemiológico descritivo do tipo série temporal. Utilizou-se informações dos casos confirmados e notificados ao Ministério da Saúde/SVS através do Sistema de Informação de Agravos de Notificação (SINANNET), do departamento de informática do Sistema Único de Saúde do Brasil (DATASUS).

Os dados foram trabalhados no programa Microsoft Excel 2019 com análise estatística e descritiva. Foram coletados os seguintes dados dos pacientes acometidos por LV: sexo, faixa etária, local de residência, óbitos e média de permanência hospitalar. Os cálculos referentes ao coeficiente de incidência da população por 100.000 habitantes foram obtidos do Instituto Brasileiro de Geografia e Estatística (IBGE) (2010). A pesquisa seguiu todos os princípios éticos, já que, por se tratar de dados secundários ao SINAN, não houve a obrigatoriedade de submissão ao Comitê de Ética em pesquisa.

\section{RESULTADOS}

No período de janeiro de 2010 até dezembro de 2019 foram contabilizados um total de 24.257 internações por Leishmaniose Visceral em todo o país, com uma média de 2.425,7 casos por ano. Divididos por regiões, 
temos 13350 (55,03\%) na região Nordeste, $4662(19,21 \%)$ na região Sudeste, 4280 (17,64\%) na região Norte, 1903 (7,84\%) na região Centro-Oeste e 62 (0,25\%) na região Sul.

Realizando uma estratificação em média anual de casos para cada região, obtivemos: Região Norte: Rondônia 0,9; Acre 0,4; Amazonas 0,9; Roraima 17,4; Pará 207,3; Amapá 0,6; Tocantins 200,5. Região Nordeste: Maranhão 359,9; Piauí 180,3; Ceará 299,5; Rio Grande do Norte 64,4; Paraíba 35,2; Pernambuco 94,4; Alagoas 32,6; Sergipe 61,8; Bahia 206,9. Região Sudeste: Minas Gerais 330; Espírito Santo 4,3; Rio de Janeiro 4,1; São Paulo 127,8. Região Sul: Paraná 2; Santa Catarina 1,9; Rio Grande do Sul 2,3. Região Centro-Oeste: Mato Grosso do Sul 109,6; Mato Grosso 23,2; Goiás 41,8; Distrito Federal 15,7

Posteriormente, com o auxílio dos dados populacionais do último censo demográfico do IBGE em 2010, obtivemos os resultados da incidência média anual da Leishmaniose visceral em cada estado.

Região Norte: Rondônia 0,05; Acre 0,05; Amazonas 0,02; Roraima 3,86; Pará 2,73; Amapá 0,08; Tocantins 14,49. Região Nordeste: Maranhão 5,47; Piauí 5,78; Ceará 3,54; Rio Grande do Norte 2,03; Paraíba 0,93; Pernambuco 1,07; Alagoas 1,04; Sergipe 2,98; Bahia 1,47. Região Sudeste: Minas Gerais 1,68; Espírito Santo 0,12; Rio de Janeiro 0,02; São Paulo 0,30.

Região Sul: Paraná 0,01; Santa Catarina 0,03; Rio Grande do Sul 0,02. Região Centro-Oeste: Mato Grosso do Sul 4,47; Mato Grosso 0,76; Goiás 0,69; Distrito Federal 0,61.

A Tabela 1 explanou o total de casos LV por ano em cada estado brasileiro, como também o total de casos por região. A região Nordeste ganhou destaque com o maior número de casos e corresponde a 55,03\% deles no período estudado. Já a região Sul apresentou um destaque positivo por corresponder ao menor número de casos $(0,25 \%)$. 
Tabela 1 - Total de casos/ano nos estados brasileiros.

\begin{tabular}{|c|c|c|c|c|c|c|c|c|c|c|c|}
\hline $\begin{array}{l}\text { Região/Unidade } \\
\text { da Federação }\end{array}$ & 2010 & 2011 & 2012 & 2013 & 2014 & 2015 & 2016 & 2017 & 2018 & 2019 & Total \\
\hline Região Norte & 416 & 538 & 447 & 379 & 308 & 321 & 353 & 432 & 623 & 463 & 4280 \\
\hline Rondônia & - & 1 & 1 & 3 & - & - & 1 & - & 1 & 2 & 9 \\
\hline Acre & - & - & 1 & 1 & 1 & 1 & - & - & - & - & 4 \\
\hline Amazonas & - & - & 1 & 2 & - & - & - & 1 & 1 & 4 & 9 \\
\hline Roraima & 11 & 9 & 7 & 12 & 17 & 22 & 28 & 38 & 12 & 18 & 174 \\
\hline Pará & 161 & 212 & 179 & 167 & 142 & 167 & 152 & 260 & 382 & 251 & 2073 \\
\hline Amapá & 1 & - & 3 & - & 2 & - & - & - & - & - & 6 \\
\hline Tocantins & 243 & 316 & 255 & 194 & 146 & 131 & 172 & 133 & 227 & 188 & 2005 \\
\hline $\begin{array}{l}\text { Região } \\
\text { Nordeste }\end{array}$ & 1150 & 1215 & 1025 & 1311 & 1696 & 1555 & 1207 & 1416 & 1548 & 1227 & 13350 \\
\hline Maranhão & 217 & 233 & 236 & 494 & 441 & 414 & 341 & 460 & 435 & 328 & 3599 \\
\hline Piauí & 169 & 161 & 146 & 183 & 213 & 225 & 173 & 174 & 218 & 141 & 1803 \\
\hline Ceará & 312 & 319 & 236 & 268 & 415 & 387 & 298 & 273 & 290 & 197 & 2995 \\
\hline $\begin{array}{l}\text { Rio Grande do } \\
\text { Norte }\end{array}$ & 73 & 81 & 68 & 56 & 78 & 56 & 55 & 49 & 64 & 64 & 644 \\
\hline Paraíba & 27 & 35 & 22 & 30 & 45 & 29 & 34 & 44 & 46 & 40 & 352 \\
\hline Pernambuco & 38 & 61 & 63 & 54 & 104 & 107 & 85 & 137 & 136 & 159 & 944 \\
\hline Alagoas & 22 & 24 & 17 & 22 & 28 & 34 & 28 & 36 & 61 & 54 & 326 \\
\hline Sergipe & 78 & 71 & 59 & 47 & 52 & 56 & 43 & 63 & 82 & 67 & 618 \\
\hline Bahia & 214 & 230 & 178 & 157 & 320 & 247 & 150 & 180 & 216 & 177 & 2069 \\
\hline Região Sudeste & 527 & 453 & 402 & 374 & 343 & 419 & 539 & 751 & 507 & 347 & 4662 \\
\hline Minas Gerais & 359 & 330 & 233 & 227 & 229 & 291 & 411 & 604 & 383 & 233 & 3300 \\
\hline Espírito Santo & 1 & 2 & 2 & - & 1 & 3 & 8 & 14 & 10 & 2 & 43 \\
\hline Rio de Janeiro & 3 & - & 3 & 8 & 5 & 3 & 3 & 6 & 3 & 7 & 41 \\
\hline São Paulo & 164 & 121 & 164 & 139 & 108 & 122 & 117 & 127 & 111 & 105 & 1278 \\
\hline Região Sul & 10 & 4 & 3 & 3 & 6 & 4 & 7 & 7 & 13 & 5 & 62 \\
\hline Paraná & 3 & 1 & 1 & 2 & 2 & 1 & 3 & 2 & 4 & 1 & 20 \\
\hline Santa Catarina & 5 & 1 & 2 & - & 1 & 2 & 2 & 3 & 1 & 2 & 19 \\
\hline $\begin{array}{l}\text { Rio Grande do } \\
\text { Sul }\end{array}$ & 2 & 2 & - & 1 & 3 & 1 & 2 & 2 & 8 & 2 & 23 \\
\hline $\begin{array}{l}\text { Região Centro- } \\
\text { Oeste }\end{array}$ & 328 & 262 & 237 & 228 & 158 & 149 & 122 & 140 & 141 & 138 & 1903 \\
\hline $\begin{array}{l}\text { Mato Grosso do } \\
\text { Sul }\end{array}$ & 225 & 157 & 163 & 141 & 96 & 77 & 52 & 68 & 55 & 62 & 1096 \\
\hline Mato Grosso & 48 & 43 & 32 & 34 & 11 & 14 & 17 & 9 & 11 & 13 & 232 \\
\hline Goiás & 38 & 39 & 27 & 37 & 33 & 43 & 37 & 50 & 60 & 54 & 418 \\
\hline Distrito Federal & 17 & 23 & 15 & 16 & 18 & 15 & 16 & 13 & 15 & 9 & 157 \\
\hline Total & 2431 & 2472 & 2114 & 2295 & 2511 & 2448 & 2228 & 2746 & 2832 & 2180 & 24257 \\
\hline
\end{tabular}

Fonte: Lima RG, et al., 2021; dados obtidos através do Ministério da Saúde e Sistema de Informações Hospitalares do Sistema Único de Saúde (SIH/SUS).

O Gráfico 1 explanou um panorama do número de casos em cada Estado brasileiro, apesar do Nordeste como região corresponder ao maior número de casos, observamos um destaque importante para Minas Gerais, Pará e Tocantins. Assim, percebe-se a heterogeneidade quanto ao acometimento entre estados de uma mesma região. 
Gráfico 1 - Número de casos por unidade da federação.

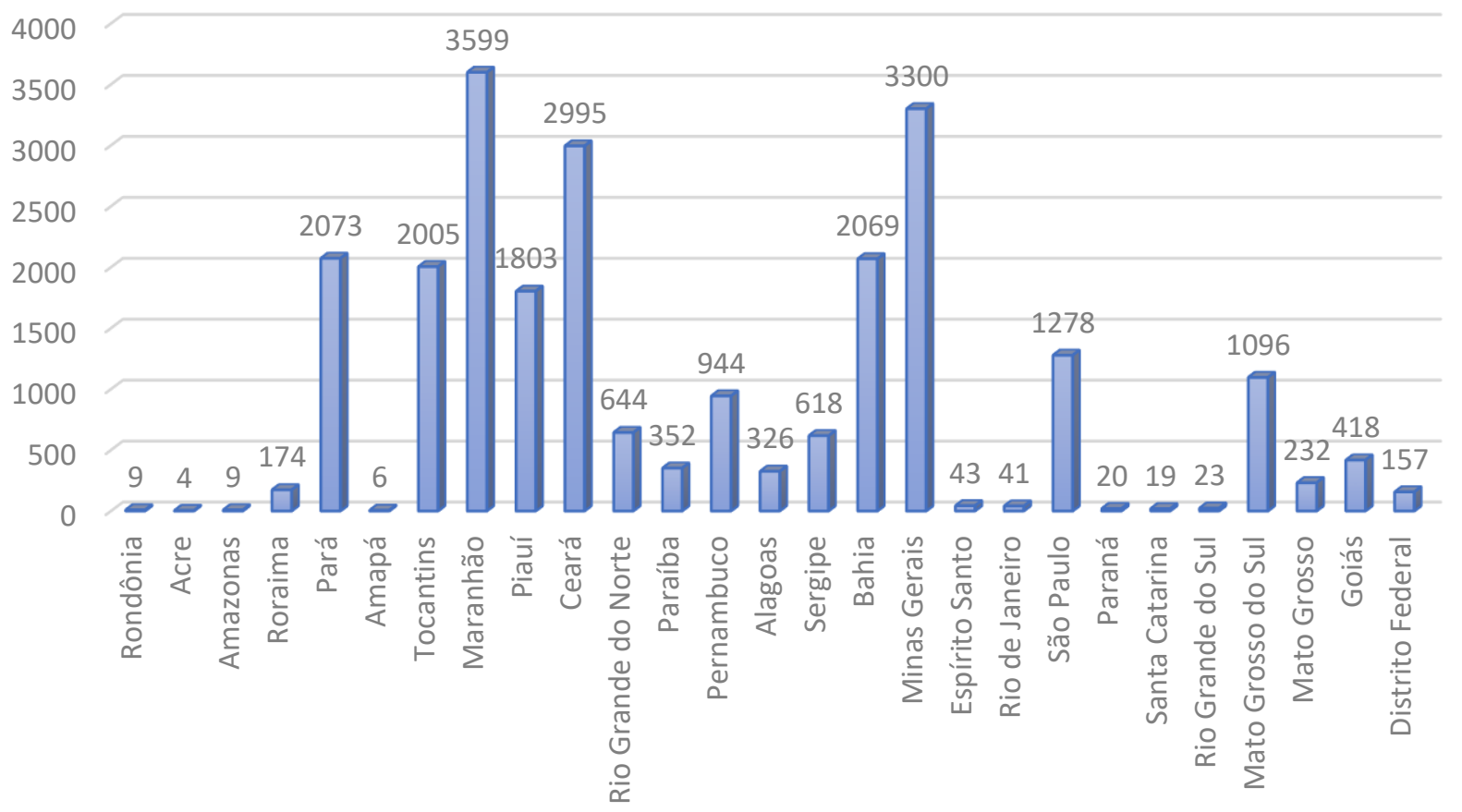

Fonte: Lima RG, et al., 2021; dados obtidos através do Ministério da Saúde e Sistema de Informações Hospitalares do Sistema Único de Saúde (SIH/SUS).

Em relação ao sexo, houve uma maior prevalência no sexo masculino que, foi responsável por um total de 15060 casos (62,08\%), enquanto o sexo feminino apresentou 9197 casos $(37,91 \%)$ (Gráfico 2).

Gráfico 2 - Incidência da leishmaniose visceral em relação ao sexo.

\section{ACOMETIMENTO POR SEXO}

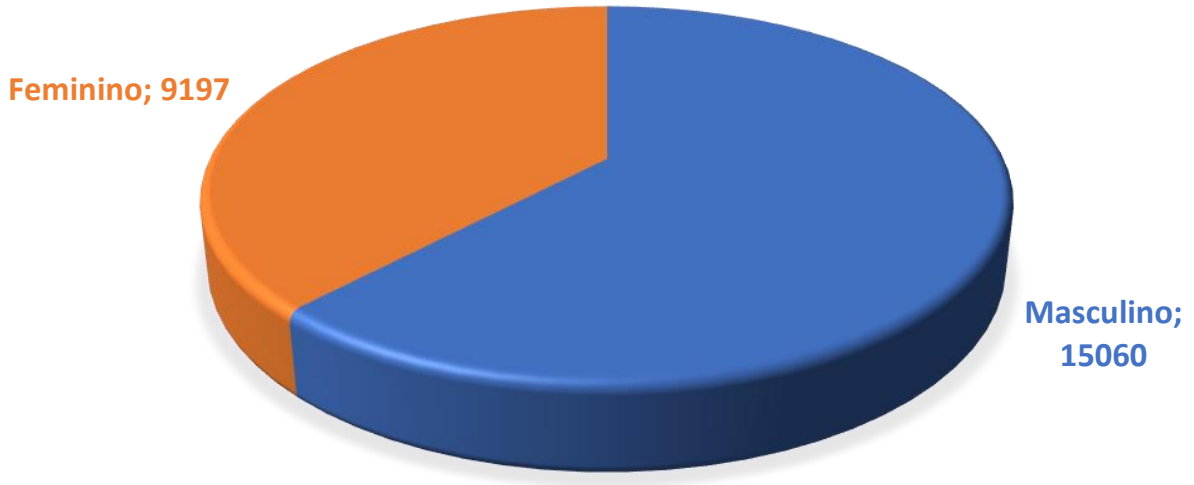

Fonte: Lima RG, et al., 2021; dados obtidos através do Ministério da Saúde e Sistema de Informações Hospitalares do Sistema Único de Saúde (SIH/SUS).

Quanto a idade, realizamos uma estratificação por faixa etária e a faixa mais acometida foi a dos 1-4 anos de idade com um total de 7537 casos (31,07\%), e a menos acometida foi a dos pacientes de 80 anos ou mais com 221 casos $(0,91 \%)$ (Tabela 2$)$. 
Tabela 2 - Total de casos em relação ao sexo e idade dos pacientes.

\begin{tabular}{|c|c|c|c|c|c|c|c|c|c|c|c|c|c|}
\hline Sexo & $\begin{array}{l}<1 \\
\text { ano }\end{array}$ & $\begin{array}{l}1 \text { a } 4 \\
\text { anos }\end{array}$ & $\begin{array}{l}5 \text { a } 9 \\
\text { anos }\end{array}$ & $\begin{array}{c}10 \text { a } \\
14 \\
\text { anos }\end{array}$ & $\begin{array}{c}15 \text { a } \\
19 \\
\text { anos }\end{array}$ & $\begin{array}{c}20 \text { a } \\
29 \\
\text { anos }\end{array}$ & $\begin{array}{c}30 \text { a } \\
39 \\
\text { anos }\end{array}$ & $\begin{array}{c}40 \text { a } \\
49 \\
\text { anos }\end{array}$ & $\begin{array}{c}50 \text { a } \\
59 \\
\text { anos }\end{array}$ & $\begin{array}{c}60 \text { a } \\
69 \\
\text { anos }\end{array}$ & $\begin{array}{c}70 \text { a } \\
79 \\
\text { anos }\end{array}$ & $\begin{array}{c}80 \\
\text { anos } \\
\mathbf{e} \\
\text { mais }\end{array}$ & Total \\
\hline Masc & 1068 & 3850 & 1307 & 751 & 752 & 1708 & 1738 & 1520 & 1094 & 761 & 369 & 142 & 15060 \\
\hline Fem & 1027 & 3687 & 1170 & 478 & 395 & 570 & 499 & 463 & 378 & 260 & 191 & 79 & 9197 \\
\hline Total & 2095 & 7537 & 2477 & 1229 & 1147 & 2278 & 2237 & 1983 & 1472 & 1021 & 560 & 221 & 24257 \\
\hline
\end{tabular}

Fonte: Lima RG, et al., 2021; dados obtidos através do Ministério da Saúde e Sistema de Informações Hospitalares do Sistema Único de Saúde (SIH/SUS).

Obtivemos um total de 1021 óbitos no período estudado, destes, $544(53,28 \%)$ na região Nordeste, 210 $(20,56 \%)$ na região Sudeste, $144(14,10 \%)$ na região Norte, $119(11,65 \%)$ na região Centro-Oeste e $4(0,39 \%)$ na região Sul. No presente estudo, a média de permanência hospitalar foi de 13,9 dias. 14,8 na região Sul, 14,5 na região Nordeste 14,1 na região Centro-Oeste, 14,1 na região Sudeste e 12,1 na região Norte.

A Tabela 3 refletiu o total de casos de LV por 100 mil habitantes em cada Estado brasileiro, isso indicou que o número de casos da doença está diretamente relacionado ao contingente populacional. O Tocantins, por exemplo, em número absoluto ocupava a $6^{a}$ posição, no entanto, após o cálculo em relação à sua população apresentou aumento relevante e passou a ocupar a $1^{\underline{a}}$ posição em número de casos.

Tabela 3 - Expressão de casos de LV por 100 mil habitantes em cada Estado.

\begin{tabular}{lc}
\hline Estados & $\begin{array}{c}\text { Casos por } \mathbf{1 0 0} \text { mil } \\
\text { habitantes }\end{array}$ \\
\hline Paraná & 0,01 \\
Rio Grande do Sul & 0,02 \\
Rio de Janeiro & 0,02 \\
Amazonas & 0,02 \\
Santa Catarina & 0,03 \\
Acre & 0,05 \\
Rondônia & 0,05 \\
Amapá & 0,08 \\
Espírito Santo & 0,12 \\
São Paulo & 0,30 \\
Distrito Federal & 0,61 \\
Goiás & 0,69 \\
Mato Grosso & 0,76 \\
Paraíba & 0,93 \\
Alagoas & 1,04 \\
Pernambuco & 1,07 \\
Bahia & 1,47 \\
Minas Gerais & 1,68 \\
Rio Grande do Norte & 2,03 \\
Pará & 2,73 \\
Sergipe & 2,98 \\
Ceará & 3,54 \\
Roraima & 3,86 \\
Mato Grosso do Sul & 4,47 \\
Maranhão & 5,47 \\
Piauí & 5,78 \\
Tocantins & 14,49 \\
\hline Fonte: Lima RG, et a &
\end{tabular}

Fonte: Lima RG, et al., 2021; dados obtidos através do Ministério da Saúde e Sistema de Informações Hospitalares do Sistema Único de Saúde (SIH/SUS). 


\section{DISCUSSÃO}

Na década de 90, aproximadamente noventa por cento (90\%) dos casos notificados de LV ocorreram na Região Nordeste. À medida que a doença se expande para as outras regiões e atinge áreas urbanas e periurbanas, esta situação vem se modificando e, no período de 2000 a 2002, a Região Nordeste já representou uma redução para 77\% dos casos do País (BRASIL, 2014). O presente estudo confirmou essa queda, já que o valor de casos encontrados foi de $55 \%$.

No entanto, em relação a incidência anual média levando em consideração o contingente populacional de cada estado, percebemos uma mudança no panorama. Maranhão que liderava o ranking com uma média de 359,9 casos por ano, passou para a terceira posição, com uma incidência média anual de aproximadamente 5,47 casos por 100.000 habitantes.

Coimbra VCS, et al. (2019), observou uma média de 255 casos por ano, no estado do Maranhão, enquadrando o município como uma zona de transmissão intensa de LV, o que corroborou com o presente estudo, já que no período analisado foi encontrada uma média de casos/ano de 355. Assim, percebeu-se que não houve mudança no perfil de transmissibilidade e epidemia da doença no estado.

Em Sergipe, foi evidenciado uma média de 61,8 casos de Leishmaniose visceral por ano, valor que deu ao estado a $12^{\text {a }}$ posição no ranking dos estados mais acometidos pela doença. Entretanto, quando analisamos a média de casos por ano em relação ao contingente populacional de cada estado, Sergipe subiu para a $7^{\underline{a}}$ posição na lista de estados com maior acometimento pela patologia. Os homens foram mais acometidos com uma proporção de $71,03 \%$ dos casos, já as mulheres, apresentaram $28,96 \%$. Foram contabilizados 22 óbitos (3,55\%). A faixa etária mais acometida no nosso estado foi a de 1 a 4 anos de idade com 126 casos (20,38\%), seguida da faixa etária de 20 a 29 anos, com 104 (16,82\%). A capital Aracaju evidenciou $74,06 \%$ dos casos em homens e $25,93 \%$ em mulheres, demonstrando uma maior concentração dos casos no sexo masculino em comparação ao Estado e à média nacional.

Santos RDF, et al. (2019), corroborou com os dados encontrados pois em seu estudo, foram evidenciados os seguintes dados: a média de casos ao ano no estado foi de 60,44. A capital Aracaju, concentrou 44,5\% dos casos do estado. Além disso, foi observada a predominância dos casos no sexo masculino (69\%) em relação ao sexo feminino (31\%).

De acordo com o nosso estudo, o estado de Sergipe apresentou do total 618 casos, um valor de $43(6,95 \%)$ casos em 2016 e, posteriormente, houve um aumento abrupto para $82(13,26 \%)$ casos em 2018, refletindo uma maior contaminação da população, ou até mesmo uma melhoria no diagnóstico e tratamento dos pacientes acometidos. Diante desse exposto, se faz necessária uma maior investigação quanto aos motivos que levaram a essa elevação importante do número de casos, a fim de criar medidas específicas para o enfrentamento dessa patologia.

Em relação ao sexo mais acometido pela doença, Coimbra VCS, et al. (2019), e Freire ML, et al. (2019), encontraram uma predominância no sexo masculino $67,45 \%$ e $66 \%$ dos casos, respectivamente. Valores semelhantes foram registrados em estudos realizados nas Américas 66,3\% (OPAS, 2018), no Rio Grande do Norte (67,09\%), Barbosa IR e Costa ICC (2013), no Piauí (66,0\%) Batista FMA, et al. (2014), e em Minas Gerais (62,6\%) Gusmão JD, et al. (2014), e em outro estudo do Piauí com $67 \%$ do sexo masculino contra $33 \%$ do sexo feminino (CORREIA AVGM, 2015).

O motivo do maior acometimento da patologia no sexo masculino ainda não é unanimidade entre os especialistas. Foi proposto por Guerra-Silveira F e Abad-Franch F (2013) que pode haver um fator hormonal ligado aos homens. Outro grupo de especialistas levantam a hipótese de uma maior exposição corporal aumentar a frequência de adoecimento nesses homens (VIANA GMC, et al., 2014; BATISTA FMA, et al., 2014). O presente estudo, corroborou com achados supracitados e encontrou uma incidência da doença no sexo masculino de $62,08 \%$ em todo o território nacional.

Lima ID, et al. (2018), afirmaram que a demografia de LV no nordeste do Brasil mudou substancialmente nos últimos 25 anos. A incidência diminuiu em crianças em associação com a melhoria da nutrição, status socioeconômico, imunizações infantis e saúde geral. Em contraste, a incidência de LV em adultos aumentou. 
O presente estudo, no entanto, apontou que a faixa etária mais acometida no Brasil foi a de 1-4 anos de idade com um total de 7537 casos $(31,07 \%)$, e a menos acometida foi a dos pacientes de 80 anos ou mais com 221 casos $(0,91 \%)$. Em concordância com o estudo de Coimbra VCS, et al. (2019), que evidenciou todas as idades foram acometidas, desde menores de 1 ano até maiores de 60 anos, com maior ocorrência na faixa etária de 0 a 4 anos.

Já a OPAS, (2018), demonstrou que as faixas etárias mais afetadas foram: $<10$ anos com $39,1 \%(1.313)$, seguido de $\geq 20<50$ anos $(34,7 \%)$ e de $>$ de 50 anos (16,96\%). No estudo de Correia AVGM (2015), no Piauí, as idades dos pacientes variaram entre um mês e 89 anos, já em Teresina, foi possível observar que a faixa etária mais frequentemente acometida na infecção por Leishmania foi aquela entre um ano e nove anos. Foi possível identificar, ainda, que existe uma frequência aumentada de casos de leishmaniose em adultos com idade entre 35 a 49 anos.

Segundo a OPAS (2018), a taxa de letalidade em 2016 foi de $7,9 \%$, considerada a maior letalidade anual registrada na região desde 2012. No período de 2012-2016 foram reportados no SisLeish um total de 1187 mortes causadas por LV representando uma média de 7,09\% de mortes. Coimbra VCS, et al. (2019), evidenciou uma taxa de letalidade global de 6,86\%. A letalidade da leishmaniose visceral entre os casos notificados pelo Piauí, entre 2007 e 2014 foi de 6,7\%, e em Teresina foi de 6,28\% (CORREIA AVGM, 2015).

O presente estudo contabilizou um total de 1021 óbitos em 10 anos, o que corresponde a 4,2\%, mostrando uma tendência a menor taxa de letalidade. Alguns dos principais fatores de risco para mortalidade foram o diagnóstico tardio e as complicações infecciosas e hemorrágicas (VIANA GMC, et al., 2014; ROCHA TJM, et al., 2015).

Sobre a permanência hospitalar, nosso estudo evidenciou uma maior permanência na região Sul, justamente a região que possui o menor número de casos registrados da patologia, o que nos levou a pensar sobre a assistência médica para a Leishmaniose visceral nessa região. Em segundo lugar, na lista de regiões com maior tempo de permanência hospitalar está a região Nordeste. A literatura carece de dados sobre a permanência hospitalar dos pacientes com Leishmaniose visceral, o que dificulta o aprofundamento e a correlação entre o adoecimento e as causas para o maior período de internamento.

\section{CONCLUSÃO}

No Brasil, vários fatores concorreram para o aparecimento de novos focos e de novas áreas endêmicas de LV, dentre eles, as transformações ambientais, a urbanização crescente e o esvaziamento rural. Apesar disso, ao longo dos anos, o maior número de casos foi mantido no Nordeste, no sexo masculino e nas crianças. Foi observado grande heterogeneidade de casos entre as cidades de um mesmo Estado, o que acabou diluindo os dados. Sergipe seguiu o padrão nacional e manteve expressivo número de casos. No entanto, destacou-se uma menor taxa de mortalidade no país, apontando-se para a importância de manter diagnóstico e tratamento precoces, e a necessidade de melhores práticas sanitárias de enfrentamento dessa patologia.

\section{REFERÊNCIAS}

1. BARBOSA IR, COSTA ICC. Aspectos clínicos e epidemiológicos da leishmaniose visceral em menores de 15 anos no estado do Rio Grande do Norte, Brasil. Scientia Médica, 2013; 23:5-11.

2. BATISTA F, et al. Leishmaniose: perfil epidemiológico dos casos notificados no estado do Piauí entre 2007 e 2011. Revista Univap, 2014; 20:44-55.

3. BRASIL. Manual de vigilância e controle da leishmaniose visceral. Brasília: Ministério da Saúde, 2014.

4. BRASIL. Guia de Vigilância em Saúde: volume único [recurso eletrônico], 3ª ed. 2019.

5. COIMBRA V, et al. Leishmaniose visceral: perfil epidemiológico dos casos notificados no município de São Luís-MA, no período de 2014 a 2017. Revista Brasileira de Educação e Saúde, 2019; 9:87-93.

6. COUTO I, et al. Visceral leishmaniasis: epidemiology and health education. Open Journal of Epidemiology and Public Health, 2018; 1: 7.

7. CORREIA AVGM. Perfil clínico - epidemiológico da leishmaniose visceral em Teresina - PI Tese (Mestrado em Medicina). Fundação Oswaldo Cruz - Fiocruz, 2015; 93. 
8. FARIAS F, et al. Perfil epidemiológico de pacientes diagnosticados com leishmaniose visceral humana no Brasil. C\&dRevista Eletrônica da Fainor, 2019; 12: 485-501.

9. FRITZSONS E, et al. Os biomas e o clima das capitais do Brasil. Revista Brasileira de Geografia Física. 2017; 10: 1152-1160.

10. FREIRE M, et al. Performance of serological tests available in Brazil for the diagnosis of human visceral leishmaniasis. Plos Neglected Tropical Diseases, Public Library of Science (PLoS), 2019; e0007484.

11. GUERRA-SILVEIRA F, ABAD-FRANCH F. Sex bias in infectious disease epidemiology: patterns and processes. PLoS One, 2013; 8.

12. GUSMÃO J, et al. Perfil epidemiológico da leishmaniose visceral no norte de Minas Gerais, Brasil, no período de 2007 a 2011. Revista Baiana de Saúde Pública, 2014; 38: 615-624.

13. LIMA I, et al. Mudança demográfica da leishmaniose visceral no nordeste do Brasil: Lições para o futuro. PLoS neglected tropical disease, 2018; 12.

14. ORGANIZAÇÃO PANAMERICANA DA SAÚDE. Leishmanioses: Informe Epidemiológico nas Américas. Washington, 2018.

15. RODRIGUES A, et al. Epidemiologia da leishmaniose visceral no município de Fortaleza, Ceará. Pesquisa Veterinária Brasileira, 2017; 37: 1119-1124.

16. ROCHA T, et al. Perfil epidemiológico relacionado aos casos de letalidade por Leishmaniose visceral em Alagoas: uma análise entre os anos de 2007 a 2012. Revista de Ciências Farmacêutica Básica e Aplicada, 2015; 36: 17-20.

17. SANTOS R, et al. Perfil epidemiológico de leishmaniose visceral em Aracaju, Sergipe. Universidade Tiradentes, 2019.

18. SILVA J, et al. Sandflies in an endemic area for Visceral Leishmaniasis in Northeastern Brazil. Rev. Bras. Parasitol. Vet., 2019; 28: 569-573.

19. UCHÔA K, et al. Vigilância epidemiológica da leishmaniose visceral: análise de indicadores e fatores ambientais associados. Revista Eletrônica Acervo Saúde, 2020; 45: 2979.

20. VIANA G, et al. Série temporal de casos de leishmaniose visceral em São Luís, Maranhão, Brasil (2001 a 2013): aspectos epidemiológicos e clínicos. Revista de Investigação Biomédica, 2014; 7: 80-90. 